International Journal of Pure and Applied Mathematics

Volume 112 No. 4 2017, 683-694

ISSN: 1311-8080 (printed version); ISSN: 1314-3395 (on-line version)

url: http://www.ijpam.eu

doi: $10.12732 /$ ijpam.v112i4.3

\title{
$L$-CLOSURE OPERATORS AND
}

\section{$L$-FUZZY PRE-PROXIMITIES}

\author{
Jung Mi Ko ${ }^{1}$, Ju-Mok $\mathrm{Oh}^{2} \S$ \\ ${ }^{1,2}$ Department of Mathematics \\ Gangneung-Wonju University \\ Gangneung, Gangwondo 210-702, KOREA
}

\begin{abstract}
In this paper, we introduce the notions of $L$-fuzzy pre-proximities and $L$-interior operators in complete residuated lattices. We obtain the $L$-fuzzy pre-proximities induced by $L$ closure operators. Moreover, we investigate the relations between the $L$-fuzzy pre-proximities and $L$-closure operators. We give their examples.
\end{abstract}

AMS Subject Classification: 03E72, 06A15, 06F07, 54A05

Key Words: complete residuated lattice, $L$-closure space, $L$-fuzzy pre-proximity space, $L$-topologies

\section{Introduction}

Hájek [7] introduced a complete residuated lattice which is an algebraic structure for many valued logic. It is an important mathematical tool for algebraic structure. By using the concepts of lower and upper approximation operators, information systems and decision rules are investigated in complete residuated lattices $[3,4,6,15,19]$. Höhle [8] introduced $L$-fuzzy topologies with algebraic structure $L$ (cqm, quantales, $M V$-algebra).

Katsaras [9-11] introduced the $L$-fuzzy proximity spaces in complete distributive lattices. Kim [13] extended the the $L$-fuzzy proximity spaces in strictly two-sided commutative quantales and investigated their topological properties.

Received: $\quad$ July 20, 2016

Revised: $\quad$ February 8, 2017

Published: February 13, 2017

$\S_{\text {Correspondence author }}$ (c) 2017 Academic Publications, Ltd. url: www.acadpubl.eu 
In this paper, we introduce the notions of $L$-fuzzy pre-proximities and $L$ closure operators in complete residuated lattices. We obtain the $L$-closure operators induced by $L$-fuzzy pre-proximities in Theorem 8 and the $L$-fuzzy preproximities induced by $L$-closure operators in Theorem 9 . Moreover, we study the relations between the $L$-fuzzy pre-proximities and $L$-closure operators. We give their examples.

\section{Preliminaries}

Definition 1. $[3,7,8]$ An algebra $(L, \wedge, \vee, \odot, \rightarrow, \perp, \top)$ is called a complete residuated lattice if it satisfies the following conditions:

(L1) $(L, \leq, \vee, \wedge, \perp, \top)$ is a complete lattice with the greatest element $\top$ and the least element $\perp$;

(L2) $(L, \odot, \top)$ is a commutative monoid;

(L3) $x \odot y \leq z$ iff $x \leq y \rightarrow z$ for $x, y, z \in L$.

In this paper, we always assume that $\left(L, \leq, \odot, \rightarrow, \oplus,{ }^{*}\right)$ is complete residuated lattice with an order reversing involution * which is defined by

$$
x \oplus y=\left(x^{*} \odot y^{*}\right)^{*}, \quad x^{*}=x \rightarrow \perp
$$

unless otherwise specified.

For $\alpha \in L, f \in L^{X}$, we denote $(\alpha \rightarrow f),(\alpha \odot f), \alpha_{X} \in L^{X}$ as $(\alpha \rightarrow f)(x)=$ $\alpha \rightarrow f(x),(\alpha \odot f)(x)=\alpha \odot f(x), \alpha_{X}(x)=\alpha$.

Lemma 2. $[3,7,8]$ For each $x, y, z, x_{i}, y_{i}, w \in L$, we have the following properties.

(1) $\top \rightarrow x=x, \perp \odot x=\perp$,

(2) If $y \leq z$, then $x \odot y \leq x \odot z, x \oplus y \leq x \oplus z, x \rightarrow y \leq x \rightarrow z$ and $z \rightarrow x \leq y \rightarrow x$

(3) $x \leq y$ iff $x \rightarrow y=\top$.

(4) $\left(\bigwedge_{i} y_{i}\right)^{*}=\bigvee_{i} y_{i}^{*},\left(\bigvee_{i} y_{i}\right)^{*}=\bigwedge_{i} y_{i}^{*}$,

(5) $x \rightarrow\left(\bigwedge_{i} y_{i}\right)=\bigwedge_{i}\left(x \rightarrow y_{i}\right)$,

(6) $\left(\bigvee_{i} x_{i}\right) \rightarrow y=\bigwedge_{i}\left(x_{i} \rightarrow y\right)$,

(7) $x \odot\left(\bigvee_{i} y_{i}\right)=\bigvee_{i}\left(x \odot y_{i}\right)$,

(8) $\left(\bigwedge_{i} x_{i}\right) \oplus y=\bigwedge_{i}\left(x_{i} \oplus y\right)$,

(9) $(x \odot y) \rightarrow z=x \rightarrow(y \rightarrow z)=y \rightarrow(x \rightarrow z)$,

(10) $x \odot y=\left(x \rightarrow y^{*}\right)^{*}$ and $x \oplus y=x^{*} \rightarrow y$,

(11) $(x \rightarrow y) \odot(z \rightarrow w) \leq(x \odot z) \rightarrow(y \odot w)$,

(12) $x \rightarrow y \leq(x \odot z) \rightarrow(y \odot z)$ and $(x \rightarrow y) \odot(y \rightarrow z) \leq x \rightarrow z$, 
(13) $(x \rightarrow y) \odot(z \rightarrow w) \leq(x \oplus z) \rightarrow(y \oplus w)$.

(14) $x \rightarrow y=y^{*} \rightarrow x^{*}$.

(15) $(x \vee y) \odot(z \vee w) \leq(x \vee z) \vee(y \odot w) \leq(x \oplus z) \vee(y \odot w)$. $\left.y_{i}\right)$,

(16) $\bigvee_{i \in \Gamma} x_{i} \rightarrow \bigvee_{i \in \Gamma} y_{i} \geq \bigwedge_{i \in \Gamma}\left(x_{i} \rightarrow y_{i}\right)$ and $\bigwedge_{i \in \Gamma} x_{i} \rightarrow \bigwedge_{i \in \Gamma} y_{i} \geq \bigwedge_{i \in \Gamma}\left(x_{i} \rightarrow\right.$

(17) $(x \odot y) \odot(z \oplus w) \leq(x \odot z) \oplus(y \odot w)$,

(18) $x \rightarrow y \leq(y \rightarrow z) \rightarrow(x \rightarrow z)$ and $x \rightarrow y \leq(z \rightarrow x) \rightarrow(z \rightarrow y)$.

Definition 3. [8] A map $\mathcal{C}: L^{X} \rightarrow L^{X}$ is called an $L$-closure operator on $X$ if $\mathcal{C}$ satisfies the following conditions:

(C1) $\mathcal{C}\left(\perp_{X}\right)=\perp_{X}$,

(C2) $\mathcal{C}(f) \geq f$, for all $f \in L^{X}$,

(C3) If $f \leq g$, then $\mathcal{C}(f) \leq \mathcal{C}(g)$ for all $f, g \in L^{X}$,

(C4) $\mathcal{C}(f \oplus g) \leq \mathcal{C}(f) \oplus \mathcal{C}(g)$.

The pair $(X, \mathcal{C})$ is called an $L$-closure space. An $L$-closure space is called topological if

(T) $\mathcal{C}(\mathcal{C}(f))=\mathcal{C}(f), \quad \forall f \in L^{X}$.

An $L$-closure space $(X, \mathcal{C})$ is said to be stratified if

(S) $\mathcal{C}(\alpha \odot f) \geq \alpha \odot \mathcal{C}(f)$.

Let $\left(X, \mathcal{C}_{X}\right)$ and $\left(Y, \mathcal{C}_{Y}\right)$ be $L$-closure spaces. $\varphi:\left(X, \mathcal{C}_{X}\right) \rightarrow\left(Y, \mathcal{C}_{Y}\right)$ is called an $L$-closure map if, for each $f \in L^{X}$,

$$
\varphi^{\rightarrow}\left(\mathcal{C}_{X}(f)\right) \leq \mathcal{C}_{Y}(\varphi \rightarrow(f)) .
$$

Definition 4. $[13,15]$ A mapping $\delta: L^{X} \times L^{X} \rightarrow L$ is called an $L$-fuzzy pre-proximity on $X$ if it satisfies the following axioms.

(P1) $\delta\left(\perp_{X}, \top_{X}\right)=\delta\left(\top_{X}, \perp_{X}\right)=\perp$.

$(\mathrm{P} 2) \delta(f, g) \geq \bigvee_{x \in X}(f(x) \odot g(x))$.

(P3) If $f_{1} \leq f_{2}$ and $g_{1} \leq g_{2}$, then $\delta\left(f_{1}, g_{1}\right) \leq \delta\left(f_{2}, g_{2}\right)$.

(P4) $\delta\left(f_{1} \odot f_{2}, g_{1} \oplus g_{2}\right) \leq \delta\left(f_{1}, g_{1}\right) \oplus \delta\left(f_{2}, g_{2}\right)$.

The pair $(X, \delta)$ is called an $L$-fuzzy pre-proximity space.

An $L$-fuzzy pre-proximity is called an $L$-fuzzy quasi-proximity on $X$ if (PQ) $\delta(f, g) \geq \bigwedge_{h}\left\{\delta(f, h) \oplus \delta\left(h^{*}, g\right)\right\}$.

An $L$-fuzzy quasi-proximity is called an $L$-fuzzy proximity on $X$ if $(\mathrm{P})$ $\delta(f, g)=\delta(g, f)$.

Let $\left(X, \delta_{1}\right)$ and $\left(Y, \delta_{2}\right)$ be $L$-fuzzy pre-proximity spaces. $\varphi:\left(X, \delta_{1}\right) \rightarrow$ $\left(Y, \delta_{2}\right)$ is called $L$-fuzzy proximity map if

$$
\delta_{1}\left(\varphi^{\leftarrow}(f), \varphi^{\leftarrow}(g)\right) \leq \delta_{2}(f, g) \forall f, g \in L^{Y} .
$$


Definition 5. [6,14] Let $X$ be a set. A mapping $R: X \times X \rightarrow L$ is called an $L$-partial order if it satisfies the following conditions:

(E1) reflexive if $R(x, x)=\top$ for all $x \in X$,

(E2) transitive if $R(x, y) \odot R(y, z) \leq R(x, z)$, for all $x, y, z \in X$,

(E3) antisymmetric if $R(x, y)=R(y, x)=\top$, then $x=y$.

Lemma 6. [6,14] For a given set $X$, define a binary mapping $S: L^{X} \times$ $L^{X} \rightarrow L$ by

$$
S(f, g)=\bigwedge_{x \in X}(f(x) \rightarrow g(x)) .
$$

Then, for each $f, g, h, k \in L^{X}$, and $\alpha \in L$, the following properties hold.

(1) $S$ is an $L$-partial order on $L^{X}$.

(2) $f \leq g$ iff $S(f, g)=\top$,

(3) If $f \leq g$, then $S(h, f) \leq S(h, g)$ and $S(f, h) \geq S(g, h)$,

(4) $S(f, g) \odot S(k, h) \leq S(f \odot k, g \odot h)$,

(5) $S(g, h) \leq S(f, g) \rightarrow S(f, h)$,

(6) $S(f, h)=\bigvee_{g \in L^{X}}(S(f, g) \odot S(g, h))$.

(7) If $\phi: X \rightarrow Y$ is a map, then for $f, g \in L^{X}$ and $h, k \in L^{Y}$,

$$
\begin{aligned}
& S(f, g) \leq S\left(\phi^{\rightarrow}(f), \phi^{\rightarrow}(g)\right), \\
& S(h, k) \leq S\left(\phi^{\leftarrow}(h), \phi^{\leftarrow}(k)\right),
\end{aligned}
$$

and the equalities hold if $\phi$ is bijective.

\section{L-Fuzzy Pre-Proximities and $L$-Closure Operators}

Lemma 7. Let $\mathcal{C}: L^{X} \rightarrow L^{X}$ a map. The following statement are equivalent.

(1) For all $f, g \in L^{X}, S(f, g) \leq S(\mathcal{C}(f), \mathcal{C}(g))$.

(2) If $f \leq g$, then $\mathcal{C}(f) \leq \mathcal{C}(g)$ and $\mathcal{C}(\alpha \odot h) \geq \alpha \odot \mathcal{C}(h)$ for all $f \in L^{X}$ and $\alpha \in L$.

(3) If $f \leq g$, then $\mathcal{C}(f) \leq \mathcal{C}(g)$ and $\mathcal{C}(\alpha \rightarrow h) \leq \alpha \rightarrow \mathcal{C}(h)$ for all $f \in L^{X}$ and $\alpha \in L$.

Proof. $(1) \Rightarrow(2)$. If $f \leq g$, then $\top=S(f, g) \leq S(\mathcal{C}(f), \mathcal{C}(g))$. Hence $\mathcal{C}(f) \leq \mathcal{C}(g)$. Put $g=\alpha \odot f$. Then $S(f, \alpha \odot f)=\alpha \leq S(\mathcal{C}(f), \mathcal{C}(\alpha \odot f))$. Hence $\alpha \odot \mathcal{C}(f) \leq \mathcal{C}(\alpha \odot f)$.

$(2) \Rightarrow(3)$. Since $\alpha \odot \mathcal{C}(\alpha \rightarrow f) \leq \mathcal{C}(\alpha \odot(\alpha \rightarrow f)) \leq \mathcal{C}(f), \mathcal{C}(\alpha \rightarrow f) \leq \alpha \rightarrow$ $\mathcal{C}(f)$. 
$(3) \Rightarrow(1)$. Since $S(f, g) \odot f \leq g$ iff $f \leq S(f, g) \rightarrow g, \mathcal{C}(f) \leq \mathcal{C}(S(f, g) \rightarrow$ $g) \leq S(f, g) \rightarrow \mathcal{C}(g)$. Hence $S(f, g) \leq S(\mathcal{C}(f), \mathcal{C}(g))$.

From the following theorem, we obtain the $L$-closure operator induced by an $L$-fuzzy pre-proximity.

Theorem 8. Let $(X, \delta)$ be an $L$-fuzzy pre-proximity space. We define a mappings $\mathcal{C}_{\delta}: L^{X} \rightarrow L^{X}$ as

$$
\mathcal{C}_{\delta}(f)=\bigwedge_{g \in L^{X}}\left(\left(S\left(f, g^{*}\right) \odot g\right) \rightarrow \delta\left(g, g^{*}\right)\right)
$$

Then $\mathcal{C}_{\delta}$ is a stratified $L$-closure operator on $X$.

Proof. (1) (C1) Since $\delta\left(\top_{X}, \perp_{X}\right)=\perp$,

$$
\begin{aligned}
& \mathcal{C}_{\delta}\left(\perp_{X}\right)=\bigwedge_{g \in L^{X}}\left(\left(S\left(\perp_{X}, g^{*}\right) \odot g\right) \rightarrow \delta\left(g, g^{*}\right)\right)\left(\text { put } g=\top_{X}\right) \\
& \left.\leq\left(S\left(\perp_{X}, \perp_{X}\right) \odot \top_{X}\right) \rightarrow \delta\left(\top_{X}, \perp_{X}\right)\right)=\perp_{X} .
\end{aligned}
$$

(C2) Since $S\left(f, g^{*}\right) \odot g=S\left(g, f^{*}\right) \odot g \leq f^{*}$, then

$$
\begin{aligned}
& \mathcal{C}_{\delta}(f)=\bigwedge_{g \in L^{X}}\left(\left(S\left(f, g^{*}\right) \odot g\right) \rightarrow \delta\left(g, g^{*}\right)\right) \geq \bigwedge_{g \in L^{X}}\left(f^{*} \rightarrow \delta\left(g, g^{*}\right)\right) \\
& =\bigwedge_{g \in L^{X}}\left(\delta^{*}\left(g, g^{*}\right) \rightarrow f\right)=\bigvee_{g \in L^{X}} \delta^{*}\left(g, g^{*}\right) \rightarrow f \geq \top \rightarrow f=f .
\end{aligned}
$$

(C4) Since

$$
\begin{aligned}
& ((a \rightarrow b) \oplus(c \rightarrow d))^{*}=(a \rightarrow b)^{*} \odot(c \rightarrow d)^{*} \\
& =\left(a \odot b^{*}\right) \odot\left(c \odot d^{*}\right)=(a \odot c) \odot\left(b^{*} \odot d^{*}\right),
\end{aligned}
$$

we have $(a \rightarrow b) \oplus(c \rightarrow d)=(a \odot c) \rightarrow(b \oplus d)$.

From Lemma 6, we obtain

$$
\begin{aligned}
& \mathcal{C}_{\delta}(f) \oplus \mathcal{C}_{\delta}(h)=\left(\bigwedge_{g \in L^{X}}\left(\left(S\left(f, g^{*}\right) \odot g\right) \rightarrow \delta\left(g, g^{*}\right)\right)\right) \\
& \oplus\left(\bigwedge_{k \in L^{X}}\left(\left(S\left(h, k^{*}\right) \odot k\right) \rightarrow \delta\left(k, k^{*}\right)\right)\right) \\
& =\bigwedge_{g, k \in L^{X}}\left(\left(S\left(f, g^{*}\right) \odot g\right) \odot\left(S\left(h, k^{*}\right) \odot k\right) \rightarrow\left(\delta\left(g, g^{*}\right) \oplus \delta\left(k, k^{*}\right)\right)\right) \\
& \geq \bigwedge_{g, k \in L^{X}}\left(\left(S\left(f \oplus h, g^{*} \oplus k^{*}\right) \odot(g \odot k)\right) \rightarrow\left(\delta\left(g \odot k, g^{*} \oplus k^{*}\right)\right)\right) \\
& \geq \mathcal{C}_{\delta}(f \oplus h) .
\end{aligned}
$$

(C3) and by Lemma $7, \mathcal{C}_{\delta}$ is stratified from: 


$$
\begin{aligned}
& S\left(\mathcal{C}_{\delta}(f), \mathcal{C}_{\delta}(h)\right) \\
& =\bigwedge_{x \in X}\left(\left(\bigwedge_{g \in L^{X}}\left(\left(S\left(f, g^{*}\right) \odot g\right) \rightarrow \delta\left(g, g^{*}\right)\right)\right)\right. \\
& \left.\rightarrow\left(\bigwedge_{k \in L^{X}}\left(\left(S\left(h, k^{*}\right) \odot k\right) \rightarrow \delta\left(k, k^{*}\right)\right)\right)\right) \\
& (\text { by Lemma } 2(16)) \\
& \geq \bigwedge_{x \in X} \bigwedge_{g \in L^{X}}\left(\left(\left(S\left(f, g^{*}\right) \odot g\right) \rightarrow \delta\left(g, g^{*}\right)\right)\right. \\
& \left.\rightarrow\left(\left(S\left(h, g^{*}\right) \odot g\right) \rightarrow \delta\left(g, g^{*}\right)\right)\right) \\
& \geq \bigwedge_{g \in L^{X}}\left(S\left(h, g^{*}\right) \odot g \rightarrow S\left(f, g^{*}\right) \odot g\right)(\text { by Lemma } 2(18)) \\
& \geq \bigwedge_{g \in L^{X}}\left(S\left(h, g^{*}\right) \rightarrow S\left(f, g^{*}\right)\right)=S(f, h) .
\end{aligned}
$$

Hence $\mathcal{C}_{\delta}$ is a stratified $L$-closure operator on $X$.

From the following theorem, we obtain the $L$-fuzzy pre-proximity induced by an $L$-closure operator.

Theorem 9. Let $(X, \mathcal{C})$ be an $L$-closure space. Define a mapping $\delta_{\mathcal{C}}$ : $L^{X} \times L^{X} \rightarrow L$ by

$$
\delta_{\mathcal{C}}(f, g)=\bigvee_{x \in X}(f(x) \odot \mathcal{C}(g)(x)) .
$$

Then we have the following properties.

(1) $\delta_{\mathcal{C}}$ is an $L$-fuzzy pre-proximity.

(2) $\delta_{\mathcal{C}}(f, g) \leq \bigwedge_{h \in L^{X}}\left(\delta_{\mathcal{C}}(f, h) \oplus \delta_{\mathcal{C}}\left(g, h^{*}\right)\right)$, the equality holds if $\mathcal{C}$ is topological.

(3) If $\mathcal{C}$ is topological, then $\delta_{\mathcal{C}}$ is an $L$-fuzzy quasi-proximity on $X$.

(4) $\mathcal{C} \leq \mathcal{C}_{\delta_{\mathcal{C}}}$, the equality holds if $\mathcal{C}$ is topological.

(5) If $\delta$ is an L-fuzzy pre-proximity on $X$, then $\delta_{\mathcal{C}_{\delta}}(f, g) \leq \bigvee_{x \in X}(f(x) \odot$ $\left.\left(g^{*}(x) \rightarrow \delta\left(g^{*}, g\right)\right)\right)$, for each $f, g \in L^{X}$.

Proof. (1) (P1) Since $\mathcal{C}\left(\perp_{X}\right)=\perp_{X}$ and $\mathcal{C}\left(\top_{X}\right)=\top_{X}$, we have

$$
\begin{aligned}
& \delta_{\mathcal{C}}\left(\perp_{X}, \top_{X}\right)=\bigvee_{x \in X}\left(\perp_{X}(x) \odot \mathcal{C}\left(\top_{X}\right)(x)\right)=\perp . \\
& \delta_{\mathcal{C}}\left(\top_{X}, \perp_{X}\right)=\bigvee_{x \in X}\left(\top_{X}(x) \odot \mathcal{C}\left(\perp_{X}\right)(x)\right)=\perp .
\end{aligned}
$$

(P2) Since $\mathcal{C}(g) \geq g$, we have

$$
\delta_{\mathcal{C}}(f, g)=\bigvee_{x \in X}(f(x) \odot \mathcal{C}(g)(x)) \geq \bigvee_{x \in X}(f(x) \odot g(x))
$$

(P3) If $g \leq g_{1}$, then $\mathcal{C}(g) \leq \mathcal{C}\left(g_{1}\right)$. Thus,

$$
\begin{aligned}
& \delta_{\mathcal{C}}(f, g)=\bigvee_{x \in X}(f(x) \odot \mathcal{C}(g)(x)) \\
& \leq \bigvee_{x \in X}\left(f_{1}(x) \odot \mathcal{C}\left(g_{1}\right)(x)\right)=\delta_{\mathcal{C}}\left(f_{1}, g_{1}\right)
\end{aligned}
$$


(P4)

$$
\begin{aligned}
& \delta_{\mathcal{C}}\left(f_{1}, g_{1}\right) \oplus \delta_{\mathcal{C}}\left(f_{2}, g_{2}\right) \\
& =\bigvee_{x \in X}\left(f_{1}(x) \odot \mathcal{C}\left(g_{1}\right)(x)\right) \oplus \bigvee_{x \in X}\left(f_{2}(x) \odot \mathcal{C}\left(g_{2}\right)(x)\right) \\
& \geq \bigvee_{x \in X}\left(\left(f_{1}(x) \odot \mathcal{C}\left(g_{1}\right)(x)\right) \oplus\left(f_{2}(x) \odot \mathcal{C}\left(g_{2}\right)(x)\right)\right) \\
& \quad(\text { by Lemma } 2(17)) \\
& \geq \bigvee_{x \in X}\left(\left(f_{1}(x) \odot f_{2}(x)\right) \odot\left(\mathcal{C}\left(g_{1}\right)(x) \oplus \mathcal{C}\left(g_{2}\right)(x)\right)\right) \\
& \geq \bigvee_{x \in X}\left(\left(f_{1}(x) \odot f_{2}(x)\right) \odot \mathcal{C}\left(g_{1} \oplus g_{2}\right)(x)\right) \\
& =\delta_{\mathcal{C}}\left(f_{1} \odot f_{2}, g_{1} \oplus g_{2}\right)
\end{aligned}
$$

Hence $\delta_{\mathcal{C}}$ is an $L$-fuzzy pre-proximity.

(2)

$$
\begin{aligned}
& \delta_{\mathcal{C}}^{*}(f, h) \odot \delta_{\mathcal{C}}^{*}\left(g, h^{*}\right) \\
& =\left(\bigvee_{x \in X}(f(x) \odot \mathcal{C}(h)(x))\right)^{*} \odot\left(\bigvee_{x \in X}\left(h^{*}(x) \odot \mathcal{C}(g)(x)\right)\right)^{*} \\
& =S\left(f, \mathcal{C}^{*}(h)\right) \odot S\left(h^{*}, \mathcal{C}^{*}(g)\right) \quad\left(\text { Since } \mathcal{C}^{*}(h) \leq h^{*},\right) \\
& \leq S\left(f, h^{*}\right) \odot S\left(h^{*}, \mathcal{C}^{*}(g)\right) \leq S\left(f, \mathcal{C}^{*}(g)\right)=\delta_{\mathcal{C}}^{*}(f, g) .
\end{aligned}
$$

Hence $\delta_{\mathcal{C}}(f, g) \leq \bigwedge_{h \in L^{X}}\left(\delta_{\mathcal{C}}(f, h) \oplus \delta_{\mathcal{C}}\left(g, h^{*}\right)\right)$.

If $\mathcal{C}$ is topological,

$$
\begin{aligned}
& \bigvee_{h \in L^{X}}\left(\delta_{\mathcal{C}}^{*}(f, h) \odot \delta_{\mathcal{C}}^{*}\left(g, h^{*}\right)\right) \\
& =\bigvee_{h \in L^{X}}\left(S\left(f, \mathcal{C}^{*}(h)\right) \odot S\left(h^{*}, \mathcal{C}^{*}(g)\right)\right) \quad(\text { Put } h=\mathcal{C}(g),) \\
& \geq S\left(f, \mathcal{C}^{*}(\mathcal{C}(g))\right) \odot S\left(\mathcal{C}^{*}(g), \mathcal{C}\left(g^{*}\right)\right) \\
& =S\left(f, \mathcal{C}^{*}(g)\right)=\delta_{\mathcal{C}}^{*}(f, g) .
\end{aligned}
$$

(3) By (2), it is trivial.

(4)

$$
\begin{aligned}
& \left.\mathcal{C}_{\delta_{\mathcal{C}}}^{*}(f)=\bigvee_{g \in L^{X}}\left(\delta_{\mathcal{C}}^{*}\left(g, g^{*}\right) \odot S\left(f, g^{*}\right) \odot g\right)\right) \\
& =\bigvee_{g \in L^{X}}\left(S\left(g, \mathcal{C}^{*}\left(g^{*}\right)\right) \odot S\left(f, g^{*}\right) \odot g\right) \\
& \leq \bigvee_{g \in L^{X}}\left(S\left(g, \mathcal{C}^{*}\left(g^{*}\right)\right) \odot S\left(\mathcal{C}(f), \mathcal{C}\left(g^{*}\right)\right) \odot g\right) \\
& =\bigvee_{g \in L^{X}}\left(S\left(g, \mathcal{C}^{*}\left(g^{*}\right)\right) \odot S\left(\mathcal{C}^{*}\left(g^{*}\right), \mathcal{C}^{*}(f)\right) \odot g\right) \\
& \leq \bigvee_{g \in L^{X}}\left(S\left(g, \mathcal{C}^{*}(f)\right) \odot g\right) \leq \mathcal{C}^{*}(f) .
\end{aligned}
$$

If $\mathcal{C}$ is topological,

$$
\begin{aligned}
& \left.\mathcal{C}_{\delta_{\mathcal{C}}}^{*}(f)=\bigvee_{g \in L^{X}}\left(\delta_{\mathcal{C}}^{*}\left(g, g^{*}\right) \odot S\left(f, g^{*}\right) \odot g\right)\right) \\
& \left(\operatorname{Put}^{*}=\mathcal{C}(f),\right) \\
& \geq S\left(\mathcal{C}^{*}(f), \mathcal{C}^{*}(\mathcal{C}(f))\right) \odot S(f, \mathcal{C}(f)) \odot \mathcal{C}^{*}(f) \\
& =S\left(\mathcal{C}^{*}(f), \mathcal{C}^{*}(f)\right) \odot S(f, \mathcal{C}(f)) \odot \mathcal{C}^{*}(f)=\mathcal{C}^{*}(f) .
\end{aligned}
$$




$$
\begin{aligned}
& \delta_{\mathcal{C}_{\delta}}(f, g)=\bigvee_{x \in X}\left(f(x) \odot \mathcal{C}_{\delta}(g)(x)\right) \\
& =\bigvee_{x \in X}\left(f(x) \odot \bigwedge_{h \in L^{X}}\left(S\left(h, g^{*}\right) \odot h(x) \rightarrow \delta\left(h, h^{*}\right)\right)\right) \\
& \leq \bigvee_{x \in X}\left(f(x) \odot\left(S\left(g^{*}, g^{*}\right) \odot g^{*}(x) \rightarrow \delta\left(g^{*}, g\right)\right)\right) \\
& =\bigvee_{x \in X}\left(f(x) \odot\left(g^{*}(x) \rightarrow \delta\left(g^{*}, g\right)\right)\right)
\end{aligned}
$$

From the following remark, we obtain the $L$-fuzzy pre-proximity induced by an $L$-topology.

Remark 10. A subset $\tau \subset L^{X}$ is called an $L$-topology (ref[8]) if (O1) $\top_{X}, \perp_{X} \in \tau$, (O2) $f \odot g \in \tau$ for $f, g \in \tau,(\mathrm{O} 3) \bigvee_{i \in \Gamma} f_{i} \in \tau$ for $\left\{f_{i} \mid i \in \Gamma\right\} \subset \tau$. Define $\mathcal{C}_{\tau}: L^{X} \rightarrow L^{X}$ as follows:

$$
\mathcal{C}_{\tau}(f)=\bigvee\left\{g \in L^{X} \mid g \geq f, g^{*} \in \tau\right\} .
$$

Then $\mathcal{C}_{\tau}$ is a topological $L$-fuzzy closure operator. By the above theorem, we obtain $\delta_{\mathcal{C}_{\tau}}$ is an $L$-fuzzy quasi-proximity with $\mathcal{C}_{\tau}=\mathcal{C}_{\delta_{\mathcal{C}_{\tau}}}$.

Theorem 11. Let $\left(X, \delta_{1}\right)$ and $\left(Y, \delta_{2}\right)$ be $L$-fuzzy pre-proximity spaces, respectively. If $\varphi:\left(X, \delta_{1}\right) \rightarrow\left(Y, \delta_{2}\right)$ is an $L$-fuzzy proximity map, then $\varphi$ : $\left(X, \mathcal{C}_{\delta_{1}}\right) \rightarrow\left(Y, \mathcal{C}_{\delta_{2}}\right)$ is an $L$-closure map.

Proof. $\varphi:\left(X, \mathcal{C}_{\delta_{1}}\right) \rightarrow\left(Y, \mathcal{C}_{\delta_{2}}\right)$ is an $L$-closure map from:

$$
\begin{aligned}
& \varphi^{\leftarrow}\left(\mathcal{C}_{\delta_{2}}(g)\right)(x)=\varphi^{\leftarrow}\left(\bigwedge_{h \in L^{Y}}\left(S\left(g, h^{*}\right) \odot h \rightarrow \delta_{2}\left(g, g^{*}\right)\right)(x)\right. \\
& =\bigwedge_{h \in L^{Y}}\left(S\left(g, h^{*}\right) \odot h(\varphi(x)) \rightarrow \delta_{2}\left(g, g^{*}\right)\right) \\
& (\text { by Lemma 6(7)) } \\
& \geq \bigwedge_{h \in L^{Y}}\left(S\left(\varphi^{\leftarrow}(g), \varphi^{\leftarrow}(h)^{*}\right) \odot \varphi^{\leftarrow}(h)(x) \rightarrow \delta_{1}\left(\varphi^{\leftarrow}(g), \varphi^{\leftarrow}(g)^{*}\right)\right) \\
& \geq \mathcal{C}_{\delta_{1}}\left(\varphi^{\leftarrow}(g)\right) .
\end{aligned}
$$

Put $g=\varphi \rightarrow(f)$. Then

$$
\begin{aligned}
& \mathcal{C}_{\delta_{1}}\left(\varphi^{\leftarrow}\left(\varphi^{\rightarrow}(f)\right)\right) \leq \varphi^{\leftarrow}\left(\mathcal{C}_{\delta_{2}}\left(\varphi^{\rightarrow}(f)\right)\right) \\
& \text { iff } \varphi^{\rightarrow}\left(\mathcal{C}_{\delta_{1}}\left(\varphi^{\leftarrow}\left(\varphi^{\rightarrow}(f)\right)\right) \leq \mathcal{C}_{\delta_{2}}\left(\varphi^{\rightarrow}(f)\right) .\right.
\end{aligned}
$$

Hence $\varphi \rightarrow\left(\mathcal{C}_{\delta_{1}}(f)\right) \leq \mathcal{C}_{\delta_{2}}(\varphi \rightarrow(f))$.

Theorem 12. Let $\left(X, \mathcal{C}_{X}\right)$ and $\left(Y, \mathcal{C}_{Y}\right)$ be $L$-closure spaces, respectively. If $\varphi:\left(X, \mathcal{C}_{X}\right) \rightarrow\left(Y, \mathcal{C}_{Y}\right)$ is an $L$-closure map, then $\varphi:\left(X, \delta_{\mathcal{C}_{X}}\right) \rightarrow\left(Y, \delta_{\mathcal{C}_{Y}}\right)$ is an $L$-fuzzy proximity map. 
Proof. Since $\varphi:\left(X, \mathcal{C}_{X}\right) \rightarrow\left(Y, \mathcal{C}_{Y}\right)$ is an $L$-closure map,

$$
\begin{aligned}
& \delta_{\mathcal{C}_{Y}}(f, g)=\bigvee_{y \in Y}\left(f(y) \odot \mathcal{C}_{Y}(g)(y)\right) \\
& \geq \bigvee_{x \in X}\left(f(\varphi(x)) \odot \mathcal{C}_{Y}(g)(\varphi(x))\right) \\
& \geq \bigvee_{x \in X}\left(\varphi^{\leftarrow}(f)(x) \odot \varphi^{\leftarrow}\left(\mathcal{C}_{Y}(g)\right)(x)\right) \\
& \left.\geq \bigvee_{x \in X}\left(\varphi^{\leftarrow}(f)(x) \odot \mathcal{C}_{X}\left(\varphi^{\leftarrow}(g)\right)\right)(x)\right) \\
& \geq \delta_{\mathcal{C}_{X}}\left(\varphi^{\leftarrow}(f), \varphi^{\leftarrow}(g)\right)
\end{aligned}
$$

Example 13. Let $\left([0,1], \odot, \oplus, \rightarrow,{ }^{*}, 0,1\right)$ be a complete residuated lattice (ref.[3,6-8]) as

$$
\begin{gathered}
x \odot y=\max \{0, x+y-1\}, x \rightarrow y=\min \{1-x+y, 1\} \\
x \oplus y=\min \{1, x+y\}, x^{*}=1-x .
\end{gathered}
$$

Let $X=\{x, y, z\}$ and $f \in[0,1]^{X}$ as follow:

$$
\begin{gathered}
f=(0.6,0.3,0.6), f \oplus f=(1,0.6,1) \\
g=(0.5,0.1,0.4), f \odot g=(0.1,0,0) . \\
f^{*}=(0.4,0.7,0.4), f^{*} \oplus f^{*}=(0.8,1,0.8) \\
g=(0.5,0.9,0.6), f^{*} \odot g^{*}=(0.9,1,1) .
\end{gathered}
$$

(1) Define a $[0,1]$-fuzzy closure operator $\mathcal{C}:[0,1]^{X} \rightarrow[0,1]^{X}$ as follows:

$$
\mathcal{C}(h)= \begin{cases}0_{X}, & \text { if } h=0_{X}, \\ f, & \text { if } h \leq f, \\ f \odot f & \text { if } h \leq f \oplus f, h \not \leq f, \\ 1_{X}, & \text { otherwise. }\end{cases}
$$

Since $\mathcal{C}$ is topological, by Theorem 9 , we obtain an [0.1]-fuzzy quasi-proximity $\delta_{\mathcal{C}}:[0,1]^{X} \times[0,1]^{X} \rightarrow[0,1]$ as follows

$$
\delta_{\mathcal{C}}(h, g)= \begin{cases}0, & \text { if } h=0_{X} \\ \bigvee_{x \in X}(h(x) \odot f(x)), & \text { or } g=0_{X} \\ \bigvee_{x \in X}(h(x) \odot(f \oplus f)(x)), & \text { if } g \leq f \\ \bigvee_{x \in X} h(x), & \text { if } g \leq f \oplus f,\end{cases}
$$

Moreover, since $\mathcal{C}$ is topological, by Theorem $9(4), \mathcal{C}_{\delta_{\mathcal{C}}}=\mathcal{C}$. 
(2) Define $\delta:[0,1]^{X} \times[0,1]^{X} \rightarrow[0,1]$ as

$$
\begin{gathered}
\delta(f, g)=\bigvee_{x \in X}(f(x) \odot g(x) \\
\quad \delta\left(f_{1}, g_{1}\right) \oplus \delta\left(f_{2}, g_{2}\right) \\
=\bigvee_{x \in X}\left(f_{1}(x) \odot g_{1}(x)\right) \oplus \bigvee_{x \in X}\left(f_{2}(x) \odot g_{2}(x)\right) \\
\geq \bigvee_{x \in X}\left(\left(f_{1}(x) \odot g_{1}(x)\right) \oplus\left(f_{2}(x) \odot g_{2}(x)\right)\right) \\
\geq \bigvee_{x \in X}\left(\left(f_{1}(x) \odot f_{2}(x)\right) \odot\left(g_{1}(x) \oplus g_{2}(x)\right)\right) \\
=\delta_{\mathcal{C}}\left(f_{1} \odot f_{2}, g_{1} \oplus g_{2}\right) \\
\bigvee_{h \in L^{X}}\left(\delta^{*}(f, h) \odot \delta^{*}\left(g, h^{*}\right)\right) \\
=\bigvee_{h \in L^{X}}\left(\bigwedge_{x \in X}\left(f(x) \rightarrow h^{*}(x)\right) \odot \bigwedge_{x \in X}\left(h^{*}(x) \rightarrow g(x)\right)\right) \\
=\bigvee_{h \in L^{X}}\left(S\left(f, h^{*}\right) \odot S\left(h^{*}, g^{*}\right)\right)=S\left(f, g^{*}\right)=\delta^{*}(f, g) . \\
\bigwedge_{h \in L^{X}}\left(\delta(f, h) \oplus \delta\left(g, h^{*}\right)\right) \\
=\left(\bigvee_{h \in L^{X}}\left(\delta^{*}(f, h) \odot \delta^{*}\left(g, h^{*}\right)\right)\right)=\delta^{*}(f, g) .
\end{gathered}
$$

Hence $\delta$ is an $[0,1]$-fuzzy quasi-proximity. Since $\bigvee_{g \in L^{X}} S\left(g, f^{*}\right) \odot g=f^{*}$, we have

$$
\begin{aligned}
& \mathcal{C}_{\delta}(f)=\bigwedge_{g \in L^{X}}\left(S\left(f, g^{*}\right) \odot g \rightarrow \delta\left(g, g^{*}\right)\right) \\
& =\left(\bigvee_{g \in L^{X}} S\left(g, f^{*}\right) \odot g\right) \rightarrow \bigvee_{x \in X}\left(g(x) \odot g^{*}(x)\right)=f^{*} \rightarrow 0=f .
\end{aligned}
$$

Thus, $\delta_{\mathcal{C}_{\delta}}=\delta$.

(3) Define a $[0,1]$-topology $\tau=\left\{0_{X}, 1_{X}, f, g, f \odot f, f \odot g\right\}$.

By Remark 10, we obtain a topological $[0,1]$-closure operator $\mathcal{C}_{\tau}:[0,1]^{X} \rightarrow$ $[0,1]^{X}$ as follows:

$$
\mathcal{C}_{\tau}(h)= \begin{cases}0_{X}, & \text { if } h=0_{X}, \\ f^{*}, & \text { if } h \leq f^{*}, \\ g^{*}, & \text { if } h \leq g^{*}, h \leq f^{*} \\ f^{*} \oplus f^{*} & \text { if } h \leq f^{*} \oplus f^{*}, h \not \leq f^{*}, h \not \leq g^{*} \\ f^{*} \oplus g^{*} & \text { if } h \leq g^{*} \oplus f^{*}, h \not \leq f^{*} \oplus f^{*} \\ 1_{X}, & \text { otherwise. }\end{cases}
$$

Since $\mathcal{C}$ is topological, by Theorem 9 , we obtain an $[0,1]$-fuzzy quasi-proximity 
$\delta_{\mathcal{C}_{\tau}}:[0,1]^{X} \times[0,1]^{X} \rightarrow[0,1]$ as follows

$$
\begin{aligned}
& \delta_{\mathcal{C}_{\tau}(h, k)} \\
& = \begin{cases}0, & \text { if } h=0_{X} \\
\bigvee_{x \in X}\left(h(x) \odot f^{*}(x)\right), & \text { or } k=0_{X} \\
\bigvee_{x \in X}\left(h(x) \odot g^{*}(x)\right), & \text { if } k \leq f^{*} \\
\bigvee_{x \in X}\left(h(x) \odot\left(f^{*} \oplus f^{*}\right)(x)\right), & \text { if } k \leq g^{*}, k \not f^{*}, \\
\bigvee_{x \in X}\left(h(x) \odot\left(f^{*} \oplus g^{*}\right)(x)\right), & \text { if } k \leq f^{*} \oplus f^{*}, k \not \leq g^{*}, \\
\bigvee_{x \in X} h(x), & \text { if } k \leq f^{*} \oplus g^{*},\end{cases} \\
&
\end{aligned}
$$

Moreover, since $\mathcal{C}$ is topological, by Theorem $9(4), \mathcal{C}_{\delta_{\mathcal{C}_{\tau}}}=\mathcal{C}$.

\section{References}

[1] G. Artico, R. Moresco, Fuzzy proximities according with Lowen fuzzy uniformities, Fuzzy Sets and Systems, 21 (1987), 85-98.

[2] R. Badard, A.A. Ramadan, A.S. Mashhour, Smooth preuniform and preproximity spaces, Fuzzy Sets and Systems, 59 (1993), 95-107.

[3] R. Bělohlávek, Fuzzy Relational Systems, Kluwer Academic Publishers, New York, 2002.

[4] D. Čimoka, A.Šostak, L-fuzzy syntopogenous structures, Part I: Fundamentals and application to $L$-fuzzy topologies, $L$-fuzzy proximities and $L$-fuzzy uniformities, Fuzzy Sets and Systems, 232 (2013), 74-97, doi: 10.1016/j.fss2013.04.009.

[5] M. El-Dardery, A.A. Ramadan, Y.C. Kim, $L$-fuzzy topogenous orders and $L$-fuzzy topologies, J. of Intelligent and Fuzzy Systems, 24, 601-609.

[6] J. Fang, I-fuzzy Alexandrov topologies and specialization orders, Fuzzy Sets and Systems, 158(2007) 2359-2374, doi: 10.1016/j.fss2007.05.001.

[7] P. Hájek, Metamathematices of Fuzzy Logic, Kluwer Academic Publishers, Dordrecht, 1998, doi: 10.1007/978-94-011-5300-3.

[8] U. Höhle, S.E. Rodabaugh, Mathematics of Fuzzy Sets: Logic, Topology, and Measure Theory, The Handbooks of Fuzzy Sets Series 3, Kluwer Academic Publishers, Boston, 1999,doi: 10.1007/978-1-4615-5079-2.

[9] A.K. Katsaras, Fuzzy proximity spaces, J. Math. Anal. Appl., 68 (1979), 100-110.

[10] A.K. Katsaras, C.G. Petalas, A unified theory of fuzzy topologies, fuzzy proximities and fuzzy uniformities, Rev. Roum. Math. Pures Appl., 28 (1983), 845-896.

[11] A. K. Katsaras, Fuzzy quasi-proximities and fuzzy quasi-uniformities, Fuzzy Sets and Systems, 27 (1988), 335-343.

[12] Y.C. Kim, Y.S. Kim, $L$-approximation spaces and $L$-fuzzy quasi-uniform spaces, Information Sciences. 179(2009), 2028-2048. 
[13] Y.C. Kim, K.C. Min, $L$-fuzzy proximities and $L$-fuzzy topologies, Information Sciences, 173 (2005), 93-113.

[14] H. Lai, D. Zhang, Fuzzy preorder and fuzzy topology, Fuzzy Sets and Systems, 157(2006) 1865-1885, doi: 10.1016/j.fss2006.02.013.

[15] A.A. Ramadan, E.H. Elkordy, Y.C. Kim, Perfect $L$-fuzzy topogenous spaces, L-fuzzy quasi-proximities and L-fuzzy quasi-uniform spaces, J. of Intelligent and Fuzzy Systems, 28 (2015), 2591-2604.

[16] A.A. Ramadan, E.H.Elkordy, Y.C. Kim, Relationships between $L$-fuzzy quasi-uniform stuctures and L-fuzzy topologies, Journal of Intelligent and Fuzzy Systems, 28 (2015), 2319-2327, doi: 10.3233/IFS-141515.

[17] S.E. Rodabaugh, E.P. Klement, Topological and Algebraic Structures In Fuzzy Sets, The Handbook of Recent Developments in the Mathematics of Fuzzy Sets, Kluwer Academic Publishers, Boston, Dordrecht, London, 2003. 